\title{
Why the Rationale for Canine Borrelia burgdorferi Vaccination Is Unpersuasive
}

\author{
Nadine A. Vogt ${ }^{1 *}$ and Christian P. G. Stevens ${ }^{2}$ \\ ${ }^{1}$ Department of Population Medicine, Ontario Veterinary College, University of Guelph, Guelph, ON, Canada, ${ }^{2}$ Department of \\ Philosophy, King's College London, London, United Kingdom
}

Keywords: Lyme, Borrelia, vaccine, canine, dog

\section{INTRODUCTION}

Canine Borrelia burgdorferi vaccines have long been a bone of contention in the veterinary profession $(1,2)$. Proponents of this non-core vaccine maintain that it is important to provide protective immunity to dogs exposed to tick-infested areas. While $B$. burgdorferi vaccines were never intended to replace tick preventives, in cases of poor compliance with product administration or product failure, pet owners can rest assured that their animal is protected against Lyme borreliosis. In the 2018 small animal consensus statement by the American College of Veterinary Internal Medicine, however, experts did not reach a consensus on whether or not to recommend the vaccine: three were in favor, and three were against (3). According to the vaccination guidelines from the American Animal Hospital Association (AAHA), not generally recommended vaccines are ones for "diseases of low clinical significance or that respond readily to treatment; vaccines for which evidence of efficacy in the field is minimal; or vaccines that may produce a relatively higher incidence of adverse events with limited benefit" (4). In this brief critical discussion, we argue that B. burgdorferi vaccines in dogs meet all of the criteria outlined by AAHA for a vaccine that is not generally recommended, and that these vaccines do not confer any clear benefit to public health, as Lyme borreliosis is a vector-borne disease. For these reasons, we conclude that the rationale for canine B. burgdorferi vaccination is unpersuasive.

\section{DISEASE OF LOW CLINICAL SIGNIFICANCE}

The vast majority of dogs appear to exhibit a kind of natural immunity to Lyme borreliosis; roughly $95 \%$ of dogs remain asymptomatic following exposure to a tick infected with B. burgdorferi (5). Although there are reports of potentially fatal cardiac and renal sequelae associated with $B$. burgdorferi in the literature (6-10), these syndromes appear to be exceedingly rare. Furthermore, these purported syndromes are challenging to conclusively link with Lyme borreliosis for that same reason (rarity of disease), as well as a lack of disease model, with no gold standard test to differentiate between clinical and incidental infections (11). It seems fair to say, then, that Lyme borreliosis meets the first criterion of AAHA of being a "disease of low clinical significance."

Received: 01 June 20

Accepted: 19 July 2021

Published: 11 August 2021

Citation:

Vogt NA and Stevens CPG (2021) Why the Rationale for Canine Borrelia

burgdorferi Vaccination is Unpersuasive.

Front. Vet. Sci. 8:719060. doi: 10.3389/fvets.2021.719060

\section{EFFECTIVE TICK PREVENTIVES AND GOOD RESPONSE TO ANTIMICROBIAL TREATMENT}

As many researchers and veterinarians in clinical practice have noted over the years, prompt treatment of clinically affected dogs with antibiotics is reported to be highly successful $(3,12)$. It would appear, then, that Lyme borreliosis is not only a "disease of low clinical significance," but it 
also "responds readily to treatment," as per the latter part of the first criterion of AAHA. Leaving dogs susceptible to tick exposures, however, is undesirable, since ticks can harbor a number of pathogenic infectious agents other than $B$. burgdorferi, such as Anaplasma, Ehrlichia, and Rickettsia spp. Tick preventives, which are shown to be highly effective in preventing the attachment and subsequent feeding of ticks (1315), are arguably the gold standard of prevention against Borrelia infection and other tick-borne diseases.

If a tick prevention regimen is properly adhered to, there should be no need for vaccination. It is occasionally argued that $B$. burgdorferi vaccines are still to be recommended in cases where owner compliance with tick preventive regimens may be an issue. A plausible rationale for recommending a vaccine, however, requires good evidence of safety and efficacy. Concerns about the safety and efficacy of $B$. burgdorferi vaccines have previously been raised, and these topics remain controversial $(2,3,10,16)$.

\section{QUESTIONABLE VACCINE EFFICACY}

Several different types of $B$. burgdorferi vaccines are currently commercially available, including several bacterins (e.g., LymeVax $^{\circledR}$, Zoetis; Nobivac ${ }^{\circledR}$ Lyme, Merck Animal Health), recombinant OspA subunit vaccines (e.g., RECOMBITEK ${ }^{\circledR}$ Lyme, Boehringer Ingelheim), and a chimeric recombinant OspA and OspC vaccine (VANGUARD ${ }^{\circledR}$ crLyme, Zoetis). To date, there are no available experimental field trials examining the efficacy of canine B. burgdorferi vaccines (17). A previous systematic review examining the efficacy of canine B. burgdorferi vaccines determined that the existing literature consisted of observational studies and challenge trials [i.e., experimental studies where disease is deliberately induced; (17)]. Notably, all three observational studies included in the systematic review suffer from a serious study design defect: none accounted for the use of tick preventives, which, by itself, could be responsible for the differences in infection rates between vaccinates and control group dogs (18-20). Thus, the second criterion of AAHA is also plausibly fulfilled: "evidence of [vaccine] efficacy in the field is minimal." It is also worth noting that B. burgdorferi vaccines are not labeled to provide protection against other species of Borrelia which are known to infect and cause clinical illness in dogs in Europe [e.g., Borrelia afzelii, Borrelia garinii; (21)].

\section{VACCINE SAFETY CONCERNS}

A retrospective observational study of over 1 million dogs demonstrated that the incidence of acute adverse events in dogs within 3 days was highest for those receiving a $B$. burgdorferi bacterin vaccine (i.e., LymeVax ${ }^{\circledR} ; 43.7 / 10,000$ doses) compared with all other vaccines examined (22). Studies evaluating the field safety of canine B. burgdorferi vaccines documenting minimal adverse events have limited generalizability, as only acute adverse events up to 10 days post-vaccination were evaluated $(19,22,23)$.
The risk of more latent adverse events has not been specifically examined for canine $B$. burgdorferi vaccines. A final consideration is that a relatively short duration of immunity necessitating annual revaccination exposes dogs to potential vaccine-related adverse events every year $(24,25)$. In conclusion, it is plausible that the third criterion of AAHA is also satisfied: B. burgdorferi "vaccines [...] may produce a relatively higher incidence of adverse events with limited benefit."

\section{DISCUSSION: A ONE HEALTH PERSPECTIVE}

Aside from the protection of the health of an individual animal, there are other good reasons for recommending a vaccine, including for the protection of public health, and/or to generate herd immunity, with the intent to limit or entirely curb the spread of disease. Canine kennel cough vaccines, for example, do not only protect the health of an individual dog but also prevent the transmission of bacteria (e.g., Bordetella) and viruses (e.g., adenovirus, parainfluenza) to other susceptible dogs within a high-density environment. This stands in contrast to B. burgdorferi, where infected dogs cannot directly transmit the infectious agent to other dogs: Lyme borreliosis is a vector-borne disease that requires a tick to carry and transmit the infectious agent. Thus, vaccination of an individual dog with a $B$. burgdorferi vaccine neither protects against direct transmission to other dogs, nor to humans. It is occasionally suggested that $B$. burgdorferi vaccines have utility in neutralizing infected ticks (26), which could, theoretically, help curb the spread of Lyme borreliosis in both humans and animals alike. In our view, the "vaccination" of ticks through their feeding on vaccinated dogs is unlikely to put any serious dent into the prevalence of $B$. burgdorferi in tick populations, since this spirochetal bacterium is geographically widespread, endemic to many regions and self-sustaining in a sylvatic transmission cycle, independent of dogs (27).

In terms of a risk-benefit analysis for the individual animal, we argue that the risks associated with B. burgdorferi vaccines are not outweighed by their benefit, as the vast majority of dogs demonstrate a natural immunity to B. burgdorferi, in contrast to other pathogenic agents. Moreover, in the subpopulation of dogs prone to developing Lyme borreliosis following exposure to $B$. burgdorferi, we cannot, at this time, rule out the possibility that this is a subpopulation of dogs with dysfunctional immune systems who may be at increased risk of having an adverse reaction to a $B$. burgdorferi vaccine (3). Future research characterizing the demographic and immunological characteristics of these canine subpopulations may shed light on this issue.

In line with AAHA guidelines, we have argued that vaccination of dogs for Lyme borreliosis is unwarranted, since clinical disease is rare, cases respond readily to treatment, alternative prevention methods are effective, and vaccine 
efficacy and safety are questionable. If this is correct, then it is in the best interests of our canine companions, and not contrary to human or canine herd health, to avoid the administration of canine $B$. burgdorferi vaccines at this time.

\section{REFERENCES}

1. Eckholm E. Caution is urged on Lyme disease vaccinations for dogs. The New York Times (1991, 22 June). Available online at: www.nytimes.com/1991/ 06/22/news/caution/is/urged/on/lyme/disease/vaccinations/for/dogs.html (accessed May 5, 2021).

2. Littman MP, Goldstein RE. Vaccinating dogs against Lyme disease: two points of view. Today's Veterinary Practice. (2014). Available online at: https:// todaysveterinarypractice.com/perspectives/vaccinating/dogs/against/lyme/ disease/two/points/of/view/ (accessed May 5, 2021).

3. Littman MP, Gerber B, Goldstein RE, Labato MA, Lappin MR, Moore GE. ACVIM consensus update on Lyme borreliosis in dogs and cats. J Vet Intern Med. (2018). 32:887-903. doi: 10.1111/jvim.15085

4. American Animal Hospital Association. Not Recommended Vaccines. AAHA Home. (2021). Available online at: www.aaha.org/aaha/guidelines/ 2020/aahaaafp/feline/vaccination/guidelines/not/recommended/vaccines/ (accessed May 5, 2021).

5. Levy SA, Magnarelli LA. Relationship between development of antibodies to Borrelia burgdorferi in dogs and the subsequent development of limb/joint borreliosis. J Am Vet Med Assoc. (1992) 200:344-7.

6. Adaszek Ł, Gatellet M, Mazurek Ł, Debiak P, Skrzypczak M, Winiarczyk S. Myocarditis secondary to Borrelia infection in a dog: a case report. Annals Parasit. (2020) 66:255-7. doi: 10.17420/ap6602.263

7. Tørnqvist-Johnsen C, Dickson SA, Rolph K, Palermo V, HodgkissGeere H, Gilmore P, et al. First report of Lyme borreliosis leading to cardiac bradydysrhythmia in two cats. JFMS Open Rep. (2020) 6:2055116919898292. doi: 10.1177/2055116919898292

8. Janus I, Noszczyk-Nowak A, Nowak M, Cepiel A, Ciaputa R, Pasławska U, et al. Myocarditis in dogs: etiology, clinical and histopathological features (11 cases: 2007-2013). Irish Vet J. (2014) 67:28. doi: 10.1186/s13620-014-0028-8

9. Littman MP. Lyme nephritis. J Vet Emerg Crit Care. (2013) 23:16373. doi: $10.1111 / \mathrm{vec} .12026$

10. Hutton TA, Goldstein RE, Njaa BL, Atwater DZ, Chang YF, Simpson KW. Search for Borrelia burgdorferi in kidneys of dogs with suspected "Lyme nephritis". J Vet Intern Med. (2008) 22:860-5. doi: 10.1111/j.1939-1676.2008.0131.x

11. Jacobson RH, Chang YF, Shin SJ. Lyme disease: Laboratory diagnosis of infected and vaccinated symptomatic dogs. Semin Vet Med Surg. (1996) 11:172-82. doi: 10.1016/s1096-2867(96)80030-2

12. Krupka I, Straubinger RK. Lyme borreliosis in dogs and cats: background, diagnosis, treatment and prevention of infections with Borrelia burgdorferi sensu stricto. Vet Clin North Am Small Anim Pract. (2010) 40:110319. doi: 10.1016/j.cvsm.2010.07.011

13. Becskei C, Liebenberg J, Thys M, Mahabir SP. Efficacy of a novel chewable tablet containing sarolaner, moxidectin and pyrantel (Simparica Trio ${ }^{\mathrm{TM}}$ ) against four common tick species infesting dogs in Europe. Parasit Vect. (2020) 13:100. doi: 10.1186/s13071-020-3949-y

14. Kondo Y, Kinoshita G, Drag M, Chester TS, Larsen D. Evaluation of the efficacy of afoxolaner against Haemaphysalis longicornis on dogs. Vet Parasitol. (2014) 201:229-31. doi: 10.1016/j.vetpar.2014.02.019

15. Fourie JJ, Luus HG, Stanneck D, Jongejan F. The efficacy of Advantix ${ }^{\circledR}$ to prevent transmission of Ehrlichia canis to dogs by Rhipicephalus sanguineus ticks. Parasite. (2013) 20:36. doi: 10.1051/parasite/201 3037

16. Dambach DM, Smith CA, Lewis RM, Van Winkle TJ. Morphologic, immunohistochemical, and ultrastructural characterization of a

\section{AUTHOR CONTRIBUTIONS}

$\mathrm{NV}$ and CS co-wrote the manuscript and contributed equally to the intellectual content. Both authors contributed to the article and approved the submitted version.

distinctive renal lesion in dogs putatively associated with Borrelia burgdorferi infection: 49 cases (1987-1992). Vet Pathol. (1997) 34:85-96. doi: 10.1177/030098589703400201

17. Vogt NA, Sargeant JM, MacKinnon MC, Versluis AM. Efficacy of Borrelia burgdorferi vaccine in dogs in North America: a systematic review and meta-analysis. J Vet Intern Med. (2019) 33:23-36. doi: 10.1111/jvim.15344

18. Levy SA, Clark KK, Glickman LT. Infection rates in dogs vaccinated and not vaccinated with an OspA Borrelia burgdorferi vaccine in a Lyme diseaseendemic area of Connecticut. Int J Appl Res Vet Med. (2005) 3:1-5.

19. Levy SA, Lissman BA, Ficke CM. Performance of a Borrelia burgdorferi bacterin in borreliosis-endemic areas. J Am Vet Med Assoc. (1993) 11:1834-8.

20. Levy SA. Use of a C6 ELISA test to evaluate the efficacy of a wholecell bacterin for the prevention of naturally transmitted canine Borrelia burgdorferi infection. Vet Ther. (2002) 3:420-4.

21. Chomel B. Lyme disease. Revue Scientifique et Technique. (2015) 34:56976. doi: 10.20506/rst.34.2.2385

22. Moore GE, Guptill LF, Ward MP, Glickman NW, Faunt KK, Lewis HB, et al. Adverse events diagnosed within three days of vaccine administration in dogs. J Am Vet Med A. (2005) 227:1102-8. doi: 10.2460/javma.2005.227.1102

23. Marconi RT, Honsberger N, Teresa Winkler M, Sobell N, King VL, Wappel S, et al. (2020). Field safety study of VANGUARD $®$ crLyme: a vaccine for the prevention of Lyme disease in dogs. Vaccine. (2005) 6:100080. doi: 10.1016/j.jvacx.2020.100080

24. LaFleur RL, Callister SM, Dant JC, Jobe DA, Lovrich SD, Warner TF, et al. One-year duration of immunity induced by vaccination with a canine Lyme disease bacterin. Clin Vaccine Immunol. (2010) 17:8704. doi: 10.1128/CVI.00524-09

25. Wikle RE, Fretwell B, Jarecki M, Jarecki-Black JC. Canine Lyme disease: oneyear duration of immunity elicited with a canine OspA monovalent Lyme vaccine. Intern J Appl Res Vet Med. (2006) 4:23-8.

26. Weese, S. COVID-19 and overdue dog/cat vaccines, part 4: Lyme disease. Worms \& Germs Blog. (2021). Available online at: www.wormsandgermsblog. com/2021/02/articles/animals/dogs/covid/and/overdue/dog/cat/vaccines/ part/4/lyme/disease/ (accessed May 5, 2021).

27. Rodríguez-Rojas JJ, Rodríguez-Moreno Á, Sánchez-Casas RM, HernándezEscareño JJ. Molecular detection of Leptospira interrogans and Borrelia burgdorferi in wild rodents from Mexico. Vector Borne Zoonotic Dis. (2020) 20:860-3. doi: 10.1089/vbz.2019.2600

Conflict of Interest: The authors declare that the research was conducted in the absence of any commercial or financial relationships that could be construed as a potential conflict of interest.

Publisher's Note: All claims expressed in this article are solely those of the authors and do not necessarily represent those of their affiliated organizations, or those of the publisher, the editors and the reviewers. Any product that may be evaluated in this article, or claim that may be made by its manufacturer, is not guaranteed or endorsed by the publisher.

Copyright (c) 2021 Vogt and Stevens. This is an open-access article distributed under the terms of the Creative Commons Attribution License (CC BY). The use, distribution or reproduction in other forums is permitted, provided the original author(s) and the copyright owner(s) are credited and that the original publication in this journal is cited, in accordance with accepted academic practice. No use, distribution or reproduction is permitted which does not comply with these terms. 